\title{
Moment conditions in strong laws of large numbers for multiple sums and random measures* $^{*}$
}

\author{
Oleg Klesov ${ }^{\dagger}$ and Ilya Molchanov ${ }^{\ddagger}$
}

\begin{abstract}
The validity of the strong law of large numbers for multiple sums $S_{\boldsymbol{n}}$ of independent identically distributed random variables $Z_{\boldsymbol{k}}, \boldsymbol{k} \leq$ $\boldsymbol{n}$, with $r$-dimensional indices is equivalent to the integrability of $|Z|\left(\log ^{+}|Z|\right)^{r-1}$, where $Z$ is the generic summand. We consider the strong law of large numbers for more general normalizations, without assuming that the summands $Z_{\boldsymbol{k}}$ are identically distributed, and prove a multiple sum generalization of the Brunk-Prohorov strong law of large numbers. In the case of identical finite moments of order $2 q$ with integer $q \geq 1$, we show that the strong law of large numbers holds with the normalization $\left(n_{1} \cdots n_{r}\right)^{1 / 2}\left(\log n_{1} \cdots \log n_{r}\right)^{1 /(2 q)+\varepsilon}$ for any $\varepsilon>0$.

The obtained results are also formulated in the setting of ergodic theorems for random measures, in particular those generated by marked point processes.
\end{abstract}

\footnotetext{
*Supported by Swiss National Science Foundation Scopes Programme Grant IZ7320_152292

${ }^{\dagger}$ Department of Mathematical Analysis and Probability Theory, National Technical University of Ukraine (KPI), Peremogy avenue, 37, Kyiv, 03056, Ukraine, klesov@matan.kpi.ua

$¥$ University of Bern, Institute of Mathematical Statistics and Actuarial Science, Sidlerstrasse 5, CH-3012 Bern, ilya.molchanov@stat.unibe.ch
} 


\section{Introduction}

Let $r \geq 1$ be an integer number and let $\mathbb{N}^{r}$ denote the set of $r$-dimensional vectors with positive integer coordinates. Elements of $\mathbb{N}^{r}$ are denoted by $\boldsymbol{k}$, $\boldsymbol{n}$ etc. The inequality $\boldsymbol{k} \leq \boldsymbol{n}$ is defined coordinatewisely, that is $k_{i} \leq n_{i}, 1 \leq$ $i \leq r$, where $\boldsymbol{k}=\left(k_{1}, \ldots, k_{r}\right)$ and $\boldsymbol{n}=\left(n_{1}, \ldots, n_{r}\right)$. Denote $|\boldsymbol{n}|=n_{1} \cdots n_{r}$. Then, $|\boldsymbol{n}| \rightarrow \infty$ means that the maximum of all coordinates of $\boldsymbol{n}$ converges to infinity and so is called max-convergence or product convergence, see [9]. Furthermore, $\boldsymbol{n} \rightarrow \infty$ means that all components of $\boldsymbol{n}$ converge to infinity, that is $\min \left(n_{1}, \ldots, n_{r}\right) \rightarrow \infty$, it is called the min-convergence in [9].

Consider an array $\left\{b_{\boldsymbol{n}}, \boldsymbol{n} \in \mathbb{N}^{r}\right\}$ of positive numbers indexed by $\mathbb{N}^{r}$ such that $b_{\boldsymbol{n}} \rightarrow \infty$ as $|\boldsymbol{n}| \rightarrow \infty$. Define partial sums of random variables $\left\{Z_{\boldsymbol{n}}, \boldsymbol{n} \in\right.$ $\left.\mathbb{N}^{r}\right\}$ by

$$
S_{\boldsymbol{n}}=\sum_{\boldsymbol{k} \leq \boldsymbol{n}} Z_{\boldsymbol{k}}, \quad \boldsymbol{n} \in \mathbb{N}^{r}
$$

The random field $\left\{Z_{\boldsymbol{n}}, \boldsymbol{n} \in \mathbb{N}^{r}\right\}$ is said to satisfy the strong law of large numbers with the normalization $\left\{b_{\boldsymbol{n}}, \boldsymbol{n} \in \mathbb{N}^{r}\right\}$ if $Z_{\boldsymbol{n}}$ is integrable for all $\boldsymbol{n}$ and

$$
\frac{1}{b_{\boldsymbol{n}}}\left(S_{\boldsymbol{n}}-\mathbf{E} S_{\boldsymbol{n}}\right) \rightarrow 0 \quad \text { a.s. as }|\boldsymbol{n}| \rightarrow \infty \text {. }
$$

If all $Z_{n}$ 's are centered or are not integrable, the validity of the strong law of large numbers means that

$$
\frac{1}{b_{n}} S_{n} \rightarrow 0 \quad \text { a.s. as }|\boldsymbol{n}| \rightarrow \infty \text {. }
$$

It is easy to see that $b_{\boldsymbol{n}}$ should grow faster than $\sqrt{\boldsymbol{n}}$. If $\left\{Z_{\boldsymbol{n}}, \boldsymbol{n} \in \mathbb{N}^{r}\right\}$ are independent copies of a centered random variable $Z$, then (2) for $b_{\boldsymbol{n}}=|\boldsymbol{n}|$ becomes the strong law of large numbers for multiple sums, which holds if and only if $\mathbf{E}\left[|Z|\left(\log ^{+}|Z|\right)^{r-1}\right]<\infty$, see [15]. Here $\log ^{+} t$ denotes the positive part of $\log t$. If $b_{\boldsymbol{n}}$ grows faster than $|\boldsymbol{n}|$, the corresponding results are variants of the Marcinkiewicz-Zygmund law. In this paper we present a whole spectrum of such results exploring relations between the strength of the moment conditions and the growth rate of the sequence of normalising constants. In particular, we show that imposing sufficiently strong moment assumptions makes it possible to bring the normalising factors to $b_{\boldsymbol{n}}=|\boldsymbol{n}|^{1 / 2}\left(\log n_{1} \cdots \log n_{r}\right)^{\varepsilon}$ for any $\varepsilon>0$.

The strong law of large numbers was used in [16] to derive the ergodic theorem for sums generated by marked point processes. We first provide an 
alternative proof (that gives a stronger result under weaker conditions) of the strong law of large numbers claimed in [16] to follow from the multivariate analogue of the Kronecker lemma. As we show in Section 4, this lemma holds only in the nonnegative case. Indeed, we provide a counterexample to a "natural" generalization of the Kronecker lemma which invalidates the proof of [16, Th. 2.1.1].

Section 2 contains several strong laws of large numbers for multiple sums of not identically distributed random variables that combine moment conditions on the summands with not so fast growing normalising constants. Along the same line, we generalize the Brunk-Prohorov criterion for the validity of the strong law of large numbers known for the case of univariate sums, see [1, 14]. In case of i.i.d. summands, the conditions simplify substantially.

Section 3 rephrases the results from Section 2 for random measures, in particularly, those generated by marked point processes.

\section{Strong laws of large numbers for multiple sums}

\subsection{Conditions on moments of order up to 2}

The field $\left\{b_{\boldsymbol{n}}, \boldsymbol{n} \in \mathbb{N}^{r}\right\}$ is said to be monotonic if $b_{\boldsymbol{k}} \leq b_{\boldsymbol{n}}$ for $\boldsymbol{k} \leq \boldsymbol{n}$ coordinatewisely. Define the increments of $\left\{b_{\boldsymbol{n}}, \boldsymbol{n} \in \mathbb{N}^{r}\right\}$ by

$$
\Delta\left[b_{\boldsymbol{n}}\right]=\sum_{\boldsymbol{m}=\left(m_{1}, \ldots, m_{r}\right) \in\{0,1\}^{r}}(-1)^{m_{1}+\cdots+m_{r}} \boldsymbol{b}_{\boldsymbol{n}-\boldsymbol{m}}
$$

where the array $\left\{b_{\boldsymbol{n}}, \boldsymbol{n} \in \mathbb{N}^{r}\right\}$ is extended for indices with non-negative coordinates by letting $b_{\boldsymbol{n}}=0$ if at least one of the coordinates of $\boldsymbol{n}$ vanishes. The non-negativity of $\Delta\left[b_{\boldsymbol{n}}\right]$ for all $\boldsymbol{n}$ is a stronger condition than the monotonicity of $\left\{b_{\boldsymbol{n}}, \boldsymbol{n} \in \mathbb{N}^{r}\right\}$.

The following Theorem 2.1 appears as [16, Th. 2.1.1] and was announced first in [15]. However, it was formulated in the particular case $\boldsymbol{n} \rightarrow \infty$ and assuming the non-negativity of increments $\Delta\left[b_{n}\right]$ for the weights. In order to deduce the strong law of large numbers from the convergence of random multiple series, it relied on the Kronecker lemma for multiple sums that was mentioned as a "simple generalization" of the univariate case in [16. p. 116]. It will be explained in Section 4 that such a generalization holds 
only assuming that the summands are non-negative, and so the proof of [16. Th. 2.1.1] was not complete. We suggest an alternative proof that derives the strong law of large numbers under the max-convergence $|\boldsymbol{n}| \rightarrow \infty$, and for this it is unavoidable to assume that

$$
b_{n} \rightarrow \infty \text { as }|\boldsymbol{n}| \rightarrow \infty
$$

instead of $\boldsymbol{n} \rightarrow \infty$ in [16]. The one-dimensional case is considered in [5].

Note that the convergence of multiple series $\sum_{n \in \mathbb{N}^{r}} a_{n}$ is always understood as the convergence of their partial sums $\sum_{\boldsymbol{k} \leq \boldsymbol{n}} a_{\boldsymbol{k}}$ as $\boldsymbol{n} \rightarrow \infty$.

Theorem 2.1. Assume that $\left\{b_{\boldsymbol{n}}, \boldsymbol{n} \in \mathbb{N}^{r}\right\}$ is monotonic. Let $\varphi$ be a positive even continuous function on $\mathbb{R}$ such that $x^{-1} \varphi(x)$ is non-decreasing and $x^{-2} \varphi(x)$ is non-increasing for $x>0$. If $\left\{Z_{\boldsymbol{n}}, \boldsymbol{n} \in \mathbb{N}^{r}\right\}$ are independent centered random variables such that

$$
\sum_{\boldsymbol{k} \in \mathbb{N}^{r}} \frac{\mathbf{E} \varphi\left(Z_{\boldsymbol{k}}\right)}{\varphi\left(b_{\boldsymbol{k}}\right)}<\infty
$$

then the series $\sum_{\boldsymbol{k} \in \mathbb{N}^{r}} Z_{\boldsymbol{k}} / b_{\boldsymbol{k}}$ converges almost surely and (11) holds.

Proof. Given the conditions imposed on $\varphi$, it follows from the proof of $[13$, Th. 6.4] that

$$
\begin{gathered}
\mathbf{P}\{|X| \geq b\} \leq \frac{\mathbf{E} \varphi(X)}{\varphi(b)}, \\
\left|\mathbf{E}\left(X \mathbf{1}_{\{|X|<b\}}\right)\right| \leq \frac{b}{\varphi(b)} \mathbf{E} \varphi(X), \\
\mathbf{E}\left(X^{2} \mathbf{1}_{\{|X|<b\}}\right) \leq \frac{b^{2}}{\varphi(b)} \mathbf{E} \varphi(X) .
\end{gathered}
$$

for each centered random variable $X$ with $\mathbf{E} \varphi(X)<\infty$. Let $X^{t}$ be the truncation of a random variable $X$ at the level $t>0$, namely $X^{t}=X \mathbf{1}_{\{|X|<t\}}$. Condition (44) together with the latter three inequalities imply that the following three series

$$
\sum_{\boldsymbol{n} \in \mathbb{N}^{r}} \mathbf{P}\left\{\left|Z_{\boldsymbol{n}}\right| \geq b_{\boldsymbol{n}}\right\}, \quad \sum_{\boldsymbol{n} \in \mathbb{N}^{r}}\left|\frac{\mathbf{E} Z_{\boldsymbol{n}}^{b_{n}}}{b_{\boldsymbol{n}}}\right|, \quad \sum_{\boldsymbol{n} \in \mathbb{N}^{r}} \frac{\operatorname{var} Z_{\boldsymbol{n}}^{b_{n}}}{b_{\boldsymbol{n}}^{2}}
$$

converge. We conclude from the convergence of the first series that

$$
\mathbf{P}\left\{Z_{\boldsymbol{n}} \neq Z_{\boldsymbol{n}}^{b_{n}} \text { infinitely often }\right\}=0
$$


by the Borel-Cantelli lemma. By [9, Th. 5.7], the convergence of the second and third series implies that $\sum Z_{\boldsymbol{n}}^{b_{n}} / b_{\boldsymbol{n}}$ converges almost surely, whence $\sum Z_{n} / b_{n}$ converges almost surely in view of (6)

Further, [9, Cor. 8.1], [11, Cor. 2.1], and convergence of the third series in (5) yield

$$
\frac{1}{b_{\boldsymbol{n}}} \sum_{\boldsymbol{k} \leq \boldsymbol{n}}\left(Z_{\boldsymbol{k}}^{b_{k}}-\mathbf{E} Z_{\boldsymbol{k}}^{b_{k}}\right) \rightarrow 0 \quad \text { a.s. as }|\boldsymbol{n}| \rightarrow \infty \text {. }
$$

The convergence of the second series in (5) together with a version of the Kronecker lemma (which is of independent interest and appears as Lemma 4.1 in the last section of the paper) imply that

$$
\frac{1}{b_{n}} \sum_{\boldsymbol{k} \leq \boldsymbol{n}}\left|\mathbf{E} Z_{\boldsymbol{k}}^{b_{k}}\right| \rightarrow 0 \quad \text { as } \quad|\boldsymbol{n}| \rightarrow \infty .
$$

Combining this result with (7) and (6) yields (1).

Condition (4) is not optimal for i.i.d. $\left\{Z_{\boldsymbol{n}}, \boldsymbol{n} \in \mathbb{N}^{r}\right\}$. For instance if $b_{\boldsymbol{n}}=|\boldsymbol{n}|$, then it would require the integrability of $Z_{1}\left(\log ^{+}\left|Z_{1}\right|\right)^{r+\varepsilon}$ for some $\varepsilon>0$, whereas the optimal condition is the integrability of $Z_{1}\left(\log ^{+}\left|Z_{1}\right|\right)^{r-1}$, see [15].

The following result is obtained by letting $\varphi(t)=|t|^{\alpha}$ in Theorem 2.1

Corollary 2.2. Let $1 \leq \alpha \leq 2$. If $\left\{b_{\boldsymbol{n}}, \boldsymbol{n} \in \mathbb{N}^{r}\right\}$ is monotonic and (3) holds, $\left\{Z_{\boldsymbol{n}}, \boldsymbol{n} \in \mathbb{N}^{r}\right\}$ are independent centered random variables with $\mathbf{E}\left|Z_{\boldsymbol{n}}\right|^{\alpha}<\infty$ for all $\boldsymbol{n}$, and

$$
\sum_{\boldsymbol{n} \in \mathbb{N}^{r}} \frac{\mathrm{E}\left|Z_{\boldsymbol{n}}\right|^{\alpha}}{b_{\boldsymbol{n}}^{\alpha}}<\infty
$$

then (2) holds.

\subsection{Brunk-Prohorov theorem for multiple sums}

The following variant of the strong law of large numbers involves higher moments.

Theorem 2.3. Let $q \geq 1$ be an integer. Assume that $\left\{b_{\boldsymbol{n}}, \boldsymbol{n} \in \mathbb{N}^{r}\right\}$ is

monotonic and (3) holds, $\left\{Z_{\boldsymbol{n}}, \boldsymbol{n} \in \mathbb{N}^{r}\right\}$ are independent centered random 
variables with $\mathbf{E} Z_{\boldsymbol{n}}^{2 q}<\infty$ for all $\boldsymbol{n}$, and

$$
\sum_{n \in \mathbb{N}^{r}} \frac{a_{n}}{b_{n}^{2 q}}<\infty
$$

for

$$
a_{\boldsymbol{n}}=\Delta\left[|\boldsymbol{n}|^{q-1} \sum_{\boldsymbol{k} \leq \boldsymbol{n}} \mathbf{E} Z_{\boldsymbol{k}}^{2 q}\right]
$$

Then (2) holds.

Proof. An analogue of Doob's inequality for multiple sums [20] yields that

$$
\mathbf{E} \max _{\boldsymbol{k} \leq \boldsymbol{n}} S_{\boldsymbol{k}}^{2 q} \leq C^{\prime} \mathbf{E} S_{\boldsymbol{n}}^{2 q} \leq C|\boldsymbol{n}|^{q-1} \sum_{\boldsymbol{k} \leq \boldsymbol{n}} \mathbf{E} Z_{\boldsymbol{k}}^{2 q}
$$

for some constants $C^{\prime}$ and $C$, where the second inequality follows by iterating the Dharmadhikari-Fabian-Jogdeo inequality [4] several times in order to reduce the dimensionality of the summation index.

Without loss of generality, assume that $b_{\boldsymbol{n}} \geq 1$ for all $\boldsymbol{n} \in \mathbb{N}^{r}$. Fix $t \geq 0$ and consider $A_{t}=\left\{\boldsymbol{n} \in \mathbb{N}^{r}: b_{\boldsymbol{n}} \leq 2^{t}\right\}$. Pick $\boldsymbol{n}_{t}$ such that $A_{t} \subseteq\left\{\boldsymbol{k}: \boldsymbol{k} \leq \boldsymbol{n}_{t}\right\}$. For $\boldsymbol{k} \leq \boldsymbol{n}_{t}$, let $Z_{\boldsymbol{k}}^{*}=Z_{\boldsymbol{k}}$ if $\boldsymbol{k} \in A_{t}$ and $Z_{\boldsymbol{k}}^{*}=0$ otherwise, and denote their multiple sums by $S_{n}^{*}=\sum_{k \leq n} Z_{k}^{*}$. These auxiliary random variables are needed to convert the summation domain to a rectangle. Finally, let

$$
a_{\boldsymbol{n}}^{*}=\Delta\left[|\boldsymbol{n}|^{q-1} \sum_{\boldsymbol{k} \leq \boldsymbol{n}} \mathbf{E}\left(Z_{\boldsymbol{k}}^{*}\right)^{2 q}\right]=\Delta\left[|\boldsymbol{n}|^{q-1} \sum_{\boldsymbol{k} \leq \boldsymbol{n}} \mathbf{E} Z_{\boldsymbol{k}}^{2 q}\right], \quad \boldsymbol{n} \in A_{t} .
$$

Note that $a_{n}^{*} \geq 0$, being the increment of the product of two monotonic fields, see [9, Lemma 8.3]. For $\boldsymbol{n} \notin A_{t}$, let $a_{\boldsymbol{n}}^{*}=0$. Reasoning as above, we obtain

$$
\mathbf{E} \max _{\boldsymbol{n} \in A_{t}} S_{\boldsymbol{n}}^{2 q} \leq \mathbf{E} \max _{\boldsymbol{n} \leq \boldsymbol{n}_{t}}\left(S_{\boldsymbol{n}}^{*}\right)^{2 q} \leq C \sum_{\boldsymbol{n} \leq \boldsymbol{n}_{t}} a_{\boldsymbol{n}}^{*}=C \sum_{\boldsymbol{n} \in A_{t}} a_{\boldsymbol{n}} .
$$

The proof is completed by referring to [9, Th. 8.3].

Remark 2.4. An analogue of Theorem 2.3 for cumulative sums, i.e. in dimension $r=1$, goes back to Brunk [1] and Prohorov [14]. They proved that, if $\zeta_{n}=\xi_{1}+\cdots+\xi_{n}$ are cumulative sums of independent random variables $\left\{\xi_{i}, i \geq 1\right\}$ and

$$
\sum_{k=1}^{\infty} \frac{\mathbf{E} \xi_{k}^{2 q}}{k^{q+1}}<\infty
$$


for $q \geq 1$, then $\left(\zeta_{n}-\mathbf{E} \zeta_{n}\right) / n \rightarrow 0$ a.s. as $n \rightarrow \infty$. The choice $q=1$ yields validity of the Kolmogorov strong law of large numbers. A similar result can be proved for a normalization by an arbitrary increasing and unbounded sequence $\left\{b_{k}, k \geq 1\right\}$ of positive numbers. Then

$$
\sum_{k=2}^{\infty} \frac{a_{k}}{b_{k}^{2 q}}<\infty
$$

substitutes (11) as a sufficient condition for the strong law of large numbers, where

$$
\begin{aligned}
a_{k} & =k^{q-1} \sum_{j=1}^{k} \mathbf{E} \xi_{j}^{2 q}-(k-1)^{q-1} \sum_{j=1}^{k-1} \mathbf{E} \xi_{j}^{2 q} \\
& =k^{q-1} \mathbf{E} \xi_{k}^{2 q}+O(1) k^{q-2} \sum_{j=1}^{k-1} \mathbf{E} \xi_{j}^{2 q} .
\end{aligned}
$$

This sequence coincides with that given by (10) in dimension $r=1$, where Theorem 2.3 becomes the Brunk-Prohorov strong law of large numbers.

For this reason, Theorem 2.3 can be called the Brunk-Prokhorov theorem for multiple sums. Other generalizations of the Brunk-Prokhorov theorem are obtained in [12] and [17].

Corollary 2.5. Assume that independent centered random variables $\left\{Z_{\boldsymbol{n}}, \boldsymbol{n} \in\right.$ $\left.\mathbb{N}^{r}\right\}$ have the same finite moment of order $2 q$. If $\left\{b_{\boldsymbol{n}}, \boldsymbol{n} \in \mathbb{N}^{r}\right\}$ is monotonic and (3) holds, then (2) follows from

$$
\sum_{n \in \mathbb{N}^{r}} \frac{|\boldsymbol{n}|^{q-1}}{b_{n}^{2 q}}<\infty
$$

In particular, (2) holds if, for some $\varepsilon>0$,

$$
b_{\boldsymbol{n}}=|\boldsymbol{n}|^{1 / 2}\left(\log n_{1} \cdots \log n_{r}\right)^{1 /(2 q)+\varepsilon} .
$$

Proof. By (10),

$$
a_{\boldsymbol{n}}=\Delta\left[|\boldsymbol{n}|^{q-1} \sum_{\boldsymbol{k} \leq \boldsymbol{n}} 1\right] \mathbf{E} Z_{1}^{2 q}=\prod_{i=1}^{r}\left(n_{i}^{q}-\left(n_{i}-1\right)^{q}\right) \mathbf{E} Z_{1}^{2 q}
$$

is of the order $|\boldsymbol{n}|^{q-1}$. 
Thus, assuming the existence of sufficiently high moments for the summands (so that $q$ becomes large), it is possible to bring the normalization to $|\boldsymbol{n}|^{1 / 2}$ times an arbitrarily small power of $\log n_{1} \cdots \log n_{r}$. If $q=1$, then condition (14) becomes the condition imposed in Corollary 2.2 with $\alpha=2$.

\subsection{Stationary case and martingale dependence}

Now assume that $\left\{Z_{\boldsymbol{n}}, \boldsymbol{n} \in \mathbb{N}^{r}\right\}$ are stationary in the wide sense, that is $\mathbf{E} Z_{\boldsymbol{n}}=0$ for all $\boldsymbol{n}, Z_{\boldsymbol{n}}$ is square integrable, and $\mathbf{E}\left[Z_{\boldsymbol{n}+\boldsymbol{k}} Z_{\boldsymbol{n}}\right]=\mathbf{E}\left[Z_{\boldsymbol{k}} Z_{0}\right]=$ $R(\boldsymbol{k})$ for all $\boldsymbol{n}, \boldsymbol{k} \in \mathbb{N}^{r}$. Then

$$
\sum_{n \in \mathbb{N}^{r}} \frac{|R(\boldsymbol{n})|}{|\boldsymbol{n}|^{2}}\left(\log n_{1}\right)^{2} \cdots\left(\log n_{r}\right)^{2}<\infty
$$

ensures the validity of the ergodic theorem for multiple sums meaning the almost sure convergence of $S_{\boldsymbol{n}} /|\boldsymbol{n}|$ to a possibly random limit, see [6, 10].

Another possible generalization for the dependent case relies on the martingale property of the field $\left\{S_{\boldsymbol{n}}, \boldsymbol{n} \in \mathbb{N}^{r}\right\}$ meaning that the conditional expectation of $S_{\boldsymbol{n}}$ given the $\sigma$-algebra generated by $S_{\boldsymbol{k}}$ with $\boldsymbol{k} \leq \boldsymbol{m}$ equals the value of the field at the coordinatewise minimum of $\boldsymbol{n}$ and $\boldsymbol{m}$, see 21]. Then $Z_{\boldsymbol{n}}=\Delta\left[S_{\boldsymbol{n}}\right], \boldsymbol{n} \in \mathbb{N}^{r}$, is the array of multivariate martingale differences. The following result is the martingale version of Corollary 2.2.

Theorem 2.6. Let $\left\{b_{\boldsymbol{n}}, \boldsymbol{n} \in \mathbb{N}^{r}\right\}$ be monotonic and (3) hold. If $\left\{Z_{\boldsymbol{n}}, \boldsymbol{n} \in \mathbb{N}^{r}\right\}$ is such that $\left\{S_{\boldsymbol{n}}, \boldsymbol{n} \in \mathbb{N}^{r}\right\}$ is a multiparameter martingale, $\mathbf{E}\left|Z_{\boldsymbol{n}}\right|^{\alpha}<\infty$ for $\alpha \in(1,2]$ and all $\boldsymbol{n}$, and

$$
\sum_{n \in \mathbb{N}^{r}} \frac{\mathbf{E}\left|Z_{n}\right|^{\alpha}}{b_{n}^{\alpha}}<\infty
$$

then (2) holds.

Proof. Let the sets $A_{t}, t \geq 0$, and multiindices $\boldsymbol{n}_{t}, t \geq 0$, be defined as in the proof of Theorem 2.3. Fix $t \geq 0$ and let random variables $Z_{k}^{*}, \boldsymbol{k} \leq \boldsymbol{n}_{t}$, and $S_{n}^{*}, \boldsymbol{n} \leq \boldsymbol{n}_{t}$, be the same as in the proof of Theorem 2.3. The multi-index generalization of Doob's maximal inequality (see Wichura [20]) yields that

$$
\mathbf{E} \max _{\boldsymbol{n} \in A_{t}}\left|S_{\boldsymbol{n}}\right|^{\alpha} \leq \mathbf{E} \max _{\boldsymbol{n} \leq \boldsymbol{n}_{t}}\left|S_{\boldsymbol{n}}^{*}\right|^{\alpha} \leq\left(\frac{\alpha}{\alpha-1}\right)^{\alpha r} \mathbf{E}\left|S_{\boldsymbol{n}_{t}}^{*}\right|^{\alpha} .
$$


Since $S_{\boldsymbol{n}}^{*}, \boldsymbol{n} \leq \boldsymbol{n}_{t}$, is a martingale in every coordinate of $\boldsymbol{n}$ when others are fixed, von Bahr-Esseen's inequality [19] yields that

$$
\mathbf{E}\left|S_{\boldsymbol{n}_{t}}^{*}\right|^{\alpha} \leq 2^{r} \sum_{k \leq \boldsymbol{n}_{t}} \mathbf{E}\left|Z_{\boldsymbol{n}}^{*}\right|^{\alpha}=2^{r} \sum_{\boldsymbol{k} \in A_{t}} \mathbf{E}\left|Z_{\boldsymbol{n}}\right|^{\alpha}
$$

Combining the latter two inequalities, we complete the proof by referring to [9, Th. 8.2].

Remark 2.7. The case $\alpha=1$ can also be treated in the martingale setting and the conditions involve the moments $\mathbf{E}\left[\left|Z_{n}\right| \log ^{+}\left|Z_{\boldsymbol{n}}\right|\right]$. This is explained by the different form of the Doob's inequality for first moments of multiple sums, see [20], that includes a logarithmic term.

\section{Ergodic theorems for random measures and point processes}

Let $S(\cdot)$ be a random measure defined on Borel sets in $\mathbb{R}^{r}$, see [3, Def. 9.1.VI]. The random measure is called stationary if $S(\cdot)$ coincides in distribution with $S(\cdot+\boldsymbol{x})$ for each translation $\boldsymbol{x} \in \mathbb{R}^{r}$. In this case, $\mathbf{E} S(\cdot)$ (if finite) is a translation invariant Borel measure on $\mathbb{R}^{r}$ and so is proportional to the Lebesgue measure $\lambda$. The random measure $S$ is called completely random if its values on disjoint sets are independent.

\subsection{Stationary random measures}

Denote by $\mathbb{I}$ the semi-open unit cube $(0,1]^{r}$ in $\mathbb{R}^{r}$. The ergodic theorem $[3$, Th. 12.2.IV] for stationary random measures establishes that $S\left(A_{n}\right) / \lambda\left(A_{n}\right)$ converges almost surely and in $L^{1}$ to $\mathbf{E}[S(\mathbb{I}) \mid \mathcal{T}]$, where $\mathcal{T}$ is the $\sigma$-algebra of translation invariant events and $\left\{A_{n}, n \geq 1\right\}$ is any convex averaging sequence. The latter means that $A_{n}, n \geq 1$, are nested convex sets such that the diameter of the largest ball inscribed in $A_{n}$ tends to infinity.

More general averaging sequences $\left\{A_{n}, n \geq 1\right\}$ were considered in [18]. While it is rather difficult to handle general non-nested sequences of sets, the following result gives an ergodic theorem for the case of $A_{n}=[0, \boldsymbol{x}]$ being (non-nested) rectangles in $\mathbb{R}_{+}^{r}$. 
Theorem 3.1. Let $S$ be a stationary random measure such that

$$
|S(\mathbb{I})|\left(\log ^{+}|S(\mathbb{I})|\right)^{r-1}
$$

is integrable. Then

$$
\frac{S([0, \boldsymbol{x}])}{\lambda([0, \boldsymbol{x}])} \rightarrow \mathbf{E}[S(\mathbb{I}) \mid \mathcal{T}] \quad \text { a.s. as } \min \left(x_{1}, \ldots, x_{r}\right) \rightarrow \infty .
$$

Proof. The value of $S([0, \boldsymbol{x}])$ can be bounded above and below using the integrals of $S(\mathbb{I}+u)$ over $u$ from $[0, \boldsymbol{x}]$ and $[0, \boldsymbol{x}-(1, \ldots, 1)]$, respectively. The convergence of these integrals is ensured by the Zygmund multivariate ergodic theorem [22], see also [7, Th. 10.12].

In the discrete version of Theorem 3.1, the min-convergence can be replaced by the max-convergence, that is

$$
\frac{S([0, \boldsymbol{n}])}{|\boldsymbol{n}|} \rightarrow \mathbf{E}[S(\mathbb{I}) \mid \mathcal{T}] \quad \text { a.s. as }|\boldsymbol{n}| \rightarrow \infty,
$$

see [9, Prop. A.2]. In the following, assume that the random measure is ergodic (or metrically transitive), so that $\mathcal{T}$ is trivial and we obtain the unconditional expectation as the limit. This is the case if $S$ is completely random.

We say that the random measure $S$ satisfies the strong law of large numbers with normalization $\left\{b_{\boldsymbol{n}}, \boldsymbol{n} \in \mathbb{N}^{r}\right\}$ if

$$
\frac{S([0, \boldsymbol{n}])-\mathbf{E} S([0, \boldsymbol{n}])}{b_{\boldsymbol{n}}} \rightarrow 0 \quad \text { a.s. as }|\boldsymbol{n}| \rightarrow \infty .
$$

Smythe's strong law of large numbers for multiple sums [15] implies that (16) holds for a stationary completely random measure under the same assumption on the logarithmic moment of $S(\mathbb{I})$ as in Theorem 3.1. If also E $S(A)=0$ for all Borel $A$, Corollary 2.2 yields that, if $\mathbf{E}|S(\mathbb{I})|^{\alpha}<\infty$ with $\alpha \in[1,2]$, then (17) holds if $\sum b_{n}^{-\alpha}$ converges. By Corollary 2.5, (17) also holds if $S(A)$ is (2q)-integrable with integer $q \geq 1$ and (14) is satisfied, which is then the Brunk-Prohorov theorem for stationary completely random measures.

The strong law of large numbers for second order stationary random fields with the discrete parameter (see Klesov [10, Gaposhkin [6]) can be also applied in this setting. 


\subsection{Random measures generated by marked point pro- cesses}

An important family of random measures is generated by marked point processes. Let $\eta=\left\{\left(\boldsymbol{x}_{i}, y_{i}\right), i \geq 1\right\}$ be a marked point process in $\mathbb{R}^{r}$, that is $\eta$ can be viewed as a locally finite set of pairs $\left(\boldsymbol{x}_{i}, y_{i}\right)$, where $\boldsymbol{x}_{i}$ is a point in $\mathbb{R}^{r}$ and $y_{i}$ is a real number regarded as the mark of $\boldsymbol{x}_{i}$, see [2, Def. 6.4.I]. A marked point process can be also defined as a non-marked point process in $\mathbb{R}^{r} \times \mathbb{R}$. Let

$$
S(A)=\sum_{i: \boldsymbol{x}_{i} \in A} y_{i}
$$

be the sum of marks for the points located in a Borel set $A$. So defined random measure $S$ is completely random if and only if $\left\{\boldsymbol{x}_{i}, i \geq 1\right\}$ form a Poisson process and the conditional distribution of $y_{i}$ is specified by a kernel $P\left(\cdot \mid \boldsymbol{x}_{i}\right)$, see [3, Prop. 10.1.VI]. Write $\mathbf{E}\left(y^{2} \mid \boldsymbol{x}\right)$ for the second moment of $y$ sampled from $P(\cdot \mid \boldsymbol{x})$ (assuming this moment is finite) and denote by $\Lambda$ the intensity measure of the Poisson process $\left\{\boldsymbol{x}_{i}, i \geq 1\right\}$.

The strong law of large numbers of the type (17) follows from the strong law of large numbers for the partial sums of the discrete random field $Z_{n}=$ $S\left(C_{\boldsymbol{n}}\right)$, where

$$
C_{\boldsymbol{n}}=\mathbb{I}+\boldsymbol{n}-(1, \ldots, 1), \quad \boldsymbol{n} \in \mathbb{N}^{r},
$$

are cubes partitioning $\mathbb{R}^{r}$.

Remark 3.2. The above construction of $S(A)$ follows the modern theory of point processes, see [3]. Instead of using the definition of a marked point process, Smythe [16] considered a point process $\left\{\boldsymbol{x}_{i}, i \geq 1\right\}$ and sequences of i.i.d. random variables $\left\{y_{i, n} ; i \geq 1\right\}$ which allocate marks to the points lying inside $C_{\boldsymbol{n}}$; he assumed that the marks are independent of the points $\left\{\boldsymbol{x}_{i}, i \geq 1\right\}$ and between different $C_{\boldsymbol{n}}$. Then $Z_{\boldsymbol{n}}$ becomes the sum of the marks for points $\boldsymbol{x}_{i} \in C_{\boldsymbol{n}}$. This situation is a special cases of our setting.

In view of centering involved in (17), it is possible to assume that $\mathbf{E}(y \mid \boldsymbol{x})=$ 0 for all $\boldsymbol{x}$. Then

$$
\mathbf{E} S\left(C_{\boldsymbol{n}}\right)=\mathbf{E} \sum_{i, j \geq 1} y_{i} y_{j} \mathbf{1}_{\boldsymbol{x}_{i} \in C_{\boldsymbol{n}}} \mathbf{1}_{\boldsymbol{x}_{j} \in C_{\boldsymbol{n}}}=\int_{C_{\boldsymbol{n}}} \mathbf{E}\left(y^{2} \mid \boldsymbol{x}\right) \Lambda(d \boldsymbol{x}) .
$$

Corollary 2.2 with $\alpha=2$ yields that (17) holds with a monotonic $\left\{b_{\boldsymbol{n}}, \boldsymbol{n} \in \mathbb{N}^{r}\right\}$ 
satisfying (3) if

$$
\sum_{\boldsymbol{n} \in \mathbb{N}^{r}} b_{\boldsymbol{n}}^{-2} \int_{C_{\boldsymbol{n}}} \mathbf{E}\left(y^{2} \mid \boldsymbol{x}\right) \Lambda(d \boldsymbol{x})<\infty .
$$

In particular, if the marks are independent of the positions, then this condition turns into convergence of the series $\sum b_{n}^{-2} \Lambda\left(C_{\boldsymbol{n}}\right)$. A similar reasoning applies for moments of order $(2 q)$ involved in the Brunk-Prohorov strong law of large numbers, but the conditions become less transparent.

\section{The Kronecker lemma for multiple series}

In fact, a generalization of Kronecker's lemma for $\mathbb{N}^{r}$ is valid only for nonnegative terms and thus the proof of [16, Th. 2.1.1] is not complete. We fill the gap in its proof below by proving a generalization of Kronecker's lemma for multiple sums.

Lemma 4.1. Assume that $\left\{x_{\boldsymbol{n}}, \boldsymbol{n} \in \mathbb{N}^{r}\right\}$ are non-negative numbers, and $\left\{b_{\boldsymbol{n}}, \boldsymbol{n} \in \mathbb{N}^{r}\right\}$ is monotonic and tends to infinity as $\boldsymbol{n} \rightarrow \infty$ (respectively, as $|\boldsymbol{n}| \rightarrow \infty)$. If the series

$$
\sum_{n \in \mathbb{N}^{r}} \frac{x_{n}}{b_{n}}
$$

converges, then

$$
\frac{1}{b_{n}} \sum_{\boldsymbol{k} \leq \boldsymbol{n}} x_{\boldsymbol{k}} \rightarrow 0 \quad \text { as } \boldsymbol{n} \rightarrow \infty(\text { resp. }|\boldsymbol{n}| \rightarrow \infty) .
$$

Proof. The statement for the convergence as $|\boldsymbol{n}| \rightarrow \infty$ coincides with [9, Prop. A.9]. The case of convergence as $\boldsymbol{n} \rightarrow \infty$ is literally the same until the very last line of the proof, where one refers to $b_{\boldsymbol{n}} \rightarrow \infty$ as $\boldsymbol{n} \rightarrow \infty$ rather than to $b_{\boldsymbol{n}} \rightarrow \infty$ as $|\boldsymbol{n}| \rightarrow \infty$, see also [8, Lemma 2.3.1].

For a direct argument, use the non-negativity condition to deduce that, for large $n$,

$$
\frac{1}{b_{n}} \sum_{\boldsymbol{k} \leq \boldsymbol{n}} x_{\boldsymbol{k}}=\frac{1}{b_{n}} \sum_{\boldsymbol{k} \leq n} \frac{x_{\boldsymbol{k}}}{b_{\boldsymbol{k}}} b_{\boldsymbol{k}} \leq \frac{1}{b_{\boldsymbol{n}}} \sum_{|\boldsymbol{k}| \leq n_{0}} x_{\boldsymbol{k}}+\sum_{|\boldsymbol{k}|>n_{0}} \frac{x_{\boldsymbol{k}}}{b_{\boldsymbol{k}}} b_{\boldsymbol{k}} .
$$

Remark 4.2. If $r=1$, then the non-negativity condition in Lemma 4.1 is not needed, since it coincides with the standard Kronecker lemma in this case. 
Remark 4.3. The non-negativity assumption on $x_{\boldsymbol{n}}$ is essential as the following two-dimensional example shows. Let $b_{\boldsymbol{n}}=n_{1} n_{2}$ for $r=2$. Then $\Delta\left[b_{\boldsymbol{n}}\right]=1$ for all $\boldsymbol{n}$. Define

$$
\boldsymbol{x}_{k_{1} k_{2}}= \begin{cases}-k_{2}, & k_{1}=1 \\ 2 k_{2}, & k_{1}=2 \\ 0, & k_{1}>2\end{cases}
$$

For all $n_{1} \geq 2$ and $n_{2} \geq 1$, we have

$$
\sum_{k_{1}=1}^{n_{1}} \sum_{k_{2}=1}^{n_{2}} \frac{\boldsymbol{x}_{k_{1} k_{2}}}{\boldsymbol{b}_{k_{1} k_{2}}}=0
$$

whence the double series

$$
\sum_{k_{1}, k_{2}=1}^{\infty} \frac{\boldsymbol{x}_{k_{1} k_{2}}}{\boldsymbol{b}_{k_{1} k_{2}}}
$$

converges to zero for any reasonable definition of the convergence in $\mathbb{N}^{2}$. However, the sequence

$$
\frac{1}{\boldsymbol{b}_{n_{1} n_{2}}} \sum_{k_{1}=1}^{n_{1}} \sum_{k_{2}=1}^{n_{2}} \boldsymbol{x}_{k_{1} k_{2}}=\frac{n_{2}+1}{2 n_{1}}, \quad n_{1} \geq 2, n_{2} \geq 1
$$

has no limit for any reasonable definition of the convergence of $\boldsymbol{n}$ to infinity.

\section{Acknowledgements}

This work has been supported by the Swiss National Science Foundation Scopes Programme Grant IZ7320_152292. The comments of the referees helped to eliminate occasional misprints and have led to numerous improvements in the presentation.

\section{References}

[1] H. D. Brunk. The strong law of large numbers. Duke Math. J., 15:181$195,1948$. 
[2] D. J. Daley and D. Vere-Jones. An Introduction to the Theory of Point Processes. Vol. I: Elementary Theory and Methods. Springer, New York, 2 edition, 2003.

[3] D. J. Daley and D. Vere-Jones. An Introduction to the Theory of Point Processes. Vol. II: General Theory and Structure. Springer, New York, 2 edition, 2008.

[4] S. W. Dharmadhikari, V. Fabian, and K. Jogdeo. Bounds on the moments of martingales. Ann. Math. Statist., 39:1719-1723, 1968.

[5] I. Fazekas and O. Klesov. A general approach to the strong laws of large numbers. Teor. Veroyatnost. i Primenen., 45:568-583, 2000.

[6] V. F. Gaposhkin. Multiparametric strong law of large numbers for homogeneous random fields. Uspekhi Mat. Nauk, 36(6(222)):197-198, 1981.

[7] O. Kallenberg. Foundations of Modern Probability. Springer-Verlag, New York, second edition, 2002.

[8] D. Khoshnevisan. Multiparameter Processes. Springer-Verlag, New York, 2002.

[9] O. Klesov. Limit Theorems for Multi-indexed Sums of Random Variables, volume 71. Springer, Heidelberg, 2014.

[10] O. I. Klesov. The strong law of large numbers for homogeneous random fields. Teor. Veroyatnost. i Mat. Statist., 25:29-40, 166, 1981.

[11] O. I. Klesov. A new method for the strong law of large numbers for random fields. Theory Stoch. Process., 4(1-2):122-128, 1998.

[12] Z. A. Lagodowski. Strong laws of large numbers for $\mathbb{B}$-valued random fields. Discrete Dyn. Nat. Soc., pages Art. ID 485412, 12, 2009.

[13] V. V. Petrov. Limit Theorems of Probability Theory, volume 4 of Oxford Studies in Probability. The Clarendon Press, Oxford University Press, New York, 1995.

[14] Yu. V. Prohorov. On the strong law of large numbers. Izvestiya Akad. Nauk SSSR. Ser. Mat., 14:523-536, 1950. 
[15] R. T. Smythe. Strong laws of large numbers for $r$-dimensional arrays of random variables. Ann. Probability, 1:164-170, 1973.

[16] R. T. Smythe. Ergodic properties of marked point processes in $R^{r}$. Ann. Inst. H. Poincaré. Sect. B (N.S.), 11:109-125, 1975.

[17] T. C. Son and D. H. Thang. The Brunk-Prokhorov strong law of large numbers for fields of martingale differences taking values in a Banach space. Statist. Probab. Lett., 83:1901-1910, 2013.

[18] A. A. Tempel'man. Ergodic theorems for general dynamical systems. Trudy Moskov. Mat. Obšč., 26:94-132, 1972. In Russian.

[19] B. von Bahr and C.-G. Esseen. Inequalities for $r$-th absolute moment of a sum of random variables, $1 \leq r \leq 2$. Ann. Math. Statist., 36:299-303, 1965 .

[20] M. J. Wichura. Inequalities with applications to the weak convergence of random processes with multi-dimensional time parameters. Ann. Math. Statist., 40:681-687, 1969.

[21] M. Zakai. Some classes of two-parameter martingales. Ann. Probab., 9:255-265, 1981.

[22] A. Zygmund. An individual ergodic theorem for non-commutative transformations. Acta Sci. Math. Szeged, 14:103-110, 1951. 